\title{
Relaxation time of the fermions in the magnetic field (I) - the case for relativistic fermions -
}

\author{
Aya Kagimura* \\ Department of Physics, Osaka University, Osaka560-0043, Japan \\ E-mail: kagimuradhet.phys.sci.osaka-u.ac.ip \\ Tetsuya Onogi \\ Department of Physics, Osaka University, Osaka560-0043, Japan \\ E-mail: onoqiephys.sci.osaka-u.ac.jp
}

The chiral magnetic effect (CME) is the quantum anomaly related electric charge transport phenomenon along the external magnetic field, which appears in various systems possessing chiral fermions, such as the quark-gluon plasma, condensed matter physics and astrophysics.

The magnetic field dependence of the relaxation time is needed to compare the theory and experiments quantitatively. However, the model calculation of the relaxation time has been made by Argyres and Adams only for the non-relativisitic fermion with the strong magnetic field limit[四].Our study is computation of the relaxation time for the relativistic fermions in the magnetic field, extending the work by Argyres and Adams.

34th annual International Symposium on Lattice Field Theory

24-30 July 2016

University of Southampton, UK

${ }^{*}$ Speaker. 


\section{Introduction}

Chiral anomaly, which is known as Adler-Bell-Jackiw anomaly after the discoverd in 1969, is an important concept in the field theory. The statement of the anomaly is that in the quantum field theory with fermions coupled with electromagnetic gauge field, the chiral fermion number is not conserved, and obeys the anomaly equation

$$
\frac{d N_{5}}{d t}=\frac{e^{2}}{2 \pi^{2}} \mathbf{E} \cdot \mathbf{B} .
$$

In 1983 Nielsen and Ninomiya pointed out that condensed matter systems have an effect analogous to the mechanism of the chiral anomaly[ㅁ]. They considered a band structure with two Weyl nodes which possess the opposite chirality, applying the parallel electric and magnetic field. The fermion states are quantized by the magnetic field to form Landau levels, and the fermion gets drifted by the electric field within a given level. Then the equation for chiral fermion number completely matches with eq.(미). At the same time, drifted fermions get scattered back by impurities. The balance between the drifting and the scattering determines the magnitude of the electric current measured in observation. They predicted the enhancement of the magnetoconductivity proportional to $\mathbf{E} \cdot \mathbf{B}$ caused by the chiral anomaly effect.

Their prediction had not been tested in the observation for 30 years, because the example of Weyl nodes had not been discovered in solids. The related recent interest of the topological band structure and Berry curvature change the situation. The interest of Weyl semimetals has begun with theoretical proposal by Wan et al [B] , and now several examples are theoretically predicted and experimentally observed. There are several papers which report the phenomena related to the

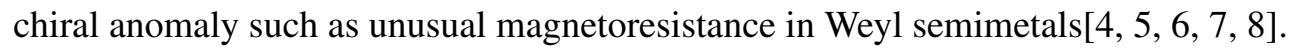

To compare these measurement and the theoretical prediction quantitatively, the magnetic field dependence of the relaxation time has to be known. The relaxation time for non-relativistic fermions in strong magnetic field limit was calculated in 1956[山]. However, since the fermion in Weyl semimetals considered here is massless fermion, the relaxation time should be for relativistic fermions. The purpose of our study is to estimate the relativistic effect to the relaxation time in strong magnetic field limit and zero temperature.

\section{Basics of the transport theory}

In this section, we derive the relation between the electric current and the relaxation time, reviewing the basics of the transport theory. The electric current density in $z$ direction is given by

$$
J=\frac{1}{V} \sum_{\mathbf{p}}(-e) \frac{\partial \varepsilon}{\partial p_{z}} f(\mathbf{p}) \times(\text { number of degeneracy points }),
$$

where $f$ is the distribution function of the fermions. Without the external field, electron states with the energy less than Fermi level energy $\mu$ are filled. The distribution function in thermodynamical equilibrium is the Fermi distribution function:

$$
f_{0}(\varepsilon)=\frac{1}{1+\exp \left(\frac{e(\mathbf{p})-\mu}{k_{B} T}\right)} .
$$


With the electric field $E$ in $z$ direction, the distribution function is slightly deviated: $f=$ $f_{0}+\delta f$. The electric field accelerates the electrons towards the same direction: $d p_{z} / d t=e E$. Therefore the imbalance of the distribution function by the drifting is

$$
\left(\frac{\partial f}{\partial t}\right)_{\mathrm{drift}}=\frac{\partial f}{\partial \mathbf{p}} \cdot \frac{d \mathbf{p}}{d t}=e E \frac{d f}{d p_{z}} .
$$

At the same time, the accelerated electrons get scattered back. So we assume that the distribution function exponentially get back into the thermodynamical equilibrium in relaxation time $\tau: \delta f \propto$ $e^{-t / \tau}$. Then the scattering effect is written as

$$
\left(\frac{\partial f}{\partial t}\right)_{\text {coll }}=-\frac{f-f_{0}}{\tau}
$$

In the lowest order in $E$, the solution of the steady state condition: $(\partial f / \partial t)_{\text {drift }}+(\partial f / \partial t)_{\text {coll }}=0$, is

$$
f=f_{0}+e E \tau \frac{\partial f_{0}}{\partial p_{z}}+\mathscr{O}\left(E^{2}\right) .
$$

From eq.(L.]), we can derive the magnitude of the electric current if we know the relaxation time.

\section{Relaxation time for relativistic fermions}

In this section we calculate the relaxation time for relativistic fermions. In this report, we take Weyl representation for $\gamma$-matrices.

\subsection{Massive Dirac fermion in magnetic field}

We consider the Dirac fermion with $m \neq 0, \mu \neq 0$, which obeys the Dirac equation:

$$
\left[i \mathbb{D}-m+\mu \gamma^{0}\right] \psi(x)=0 .
$$

Since we consider constant magnetic field in $z$ direction, we set the gauge field; $A^{2}=B x$ and the other components are zero. Multiplying $\gamma^{0}$ to the Dirac equation (B. 1 (1) from left,

$$
\left[i \partial_{0}+\mu+i \gamma^{0} \gamma^{i} D_{i}-m \gamma^{0}\right] \psi(x)=0 .
$$

To obtain the wave function $\psi(x)$, first we introduce an auxiliary function $\Phi$ which satisfies

$$
\left[i \partial_{0}+\mu+i \gamma^{0} \gamma^{i} D_{i}-m \gamma^{0}\right]\left[i \partial_{0}+\mu-i \gamma^{0} \gamma^{i} D_{i}+m \gamma^{0}\right] \Phi=0
$$

Then we can obtain the solution to eq.(B.]) for $\psi(x)$ as

$$
\psi(x)=\left[i \partial_{0}+\mu-i \gamma^{0} \gamma^{i} D_{i}+m \gamma^{0}\right] \Phi .
$$

From eq.(B.3]), taking the energy as $\omega$ and the momenta in $y, z$ directions as $p_{2}, p_{3}$, eigenfunction $\Phi$ satisfies a harmonic oscillator type equation

$$
\left[-\partial_{1}^{2}+(e B)^{2}\left(x-\frac{p_{2}}{e B}\right)^{2}+p_{3}^{2}+m^{2}-e B \sigma^{3}\right] \Phi=(\omega+\mu)^{2} \Phi .
$$


Therefore, the energy levels are given by Landau levels

$$
\omega_{n, \sigma^{3}}\left(p_{3}\right)=-\mu \pm \sqrt{2 e B\left(n+\frac{1}{2}\right)+p_{3}^{2}+m^{2}-e B \sigma^{3}} .
$$

Then we get

$$
v_{z} \equiv \frac{\partial \varepsilon}{\partial p_{z}}=\frac{p_{z}}{\varepsilon}
$$

where $\varepsilon=\omega+\mu$. The eigenfunction $\Phi$ is

$$
\begin{aligned}
\Phi_{n, \sigma^{3}, \gamma_{5}}\left(x, p_{2}, p_{3}\right)= & N_{n, \sigma^{3}, \gamma_{5}}\left(p_{3}\right) \exp \left(i p_{2} y+i p_{3} z\right) \sqrt[4]{\frac{e B}{\pi 2^{2 n} n !}} \\
& \times \exp \left[-\frac{1}{2} e B\left(x-\frac{p_{2}}{e B}\right)^{2}\right] H_{n}\left(\sqrt{e B} x-\frac{p_{2}}{\sqrt{e B}}\right) \chi_{\sigma^{3}, \gamma_{5}},
\end{aligned}
$$

where $N_{n, \sigma^{3}, \gamma_{5}}\left(p_{3}\right)$ is unknown normalization constant, $H_{n}\left(\sqrt{e B} x-\frac{p_{2}}{\sqrt{e B}}\right)$ are Hermite polynomials, and $\chi_{\sigma^{3}, \gamma_{5}}$ is the eigen spinor of $\sigma^{3}$ and $\gamma_{5}$ given by

$$
\chi_{+, L}=\left(\begin{array}{l}
1 \\
0 \\
0 \\
0
\end{array}\right), \chi_{-, L}=\left(\begin{array}{l}
0 \\
1 \\
0 \\
0
\end{array}\right), \chi_{+, R}=\left(\begin{array}{l}
0 \\
0 \\
1 \\
0
\end{array}\right), \chi_{-, R}=\left(\begin{array}{l}
0 \\
0 \\
0 \\
1
\end{array}\right) .
$$

A simple calculation shows that the eigenfunction $\Phi$ is normalized as

$$
\int d x \Phi^{\dagger} \Phi=\left|N_{n, \sigma_{3}, \gamma_{5}}\left(p_{3}\right)\right|^{2} \chi^{\dagger} \chi
$$

The wave function of the Dirac fermion is obtained as

$$
\begin{aligned}
\psi(x) & =\left\langle x \mid n, \sigma^{3} ; p_{y}, p_{z}\right\rangle \\
& =\left[\omega_{n, \sigma^{3}}\left(p_{z}\right)+\mu-i \gamma^{0} \gamma^{i} D_{i}+m \gamma^{0}\right]\left(\Phi_{n, \sigma^{3}, L}\left(x, p_{y}, p_{z}\right)+\Phi_{n, \sigma^{3} \cdot R}\left(x, p_{y}, p_{z}\right)\right) .
\end{aligned}
$$

The normalization constant $N_{n, \sigma^{3}}\left(p_{z}\right)$ is determined by the normalization condition

$$
\left\langle n^{\prime}, \sigma^{3} ; p_{y}^{\prime} \cdot p_{z}^{\prime} \mid n, \sigma^{3} ; p_{y}, p_{z}\right\rangle=(2 \pi)^{2} \delta\left(p_{y}-p_{y}^{\prime}\right) \delta\left(p_{z}-p_{z}^{\prime}\right) \delta_{n, n^{\prime}} \delta_{\sigma^{3}, \sigma^{3^{\prime}}} .
$$

In the strong magnetic limit $e B \rightarrow \infty$ only the lowest energy band $n=0, \sigma^{3}=+1$ contribute the scattering process. So we consider only the lowest energy band. After some calculation, we get the lowest energy wave function

$$
\begin{aligned}
\psi_{0,+}\left(\mathbf{x}, p_{y}, p_{z}\right)= & \left(\omega_{0,+}\left(p_{z}\right)+\mu-p_{z}+m\right) \Phi_{0,+, L}\left(x, p_{y}, p_{z}\right) \\
& +\left(\omega_{0,+}\left(p_{z}\right)+\mu+p_{z}+m\right) \Phi_{0,+, R}\left(x, p_{y}, p_{z}\right) .
\end{aligned}
$$

Then we notice

$$
\begin{aligned}
\left\langle p_{y}^{\prime} \cdot p_{z}^{\prime} \mid p_{y}, p_{z}\right\rangle & =\int d^{3} x\left\langle p_{y}^{\prime} \cdot p_{z}^{\prime} \mid x\right\rangle\left\langle x \mid p_{y}, p_{z}\right\rangle \\
& =4\left(\omega\left(p_{z}\right)+\mu\right)\left(\omega\left(p_{z}\right)+\mu+m\right)\left|N\left(p_{z}\right)\right|^{2}(2 \pi)^{2} \delta\left(p_{y}-p_{y}^{\prime}\right) \delta\left(p_{z}-p_{z}^{\prime}\right) .
\end{aligned}
$$

From the normalization condition (B.TI), the normalization constant is obtained as

$$
\left|N_{0,+}\left(p_{z}\right)\right|^{2}=\frac{1}{4\left(\omega\left(p_{z}\right)+\mu\right)\left(\omega\left(p_{z}\right)+\mu+m\right)} .
$$




\subsection{Relaxation time for scattering by charged impurities}

Since we consider only the lowest energy band $n=0, \sigma^{3}=+1$, let us omit the labels $n, \sigma^{3}$ for short.

The variation of the distribution function by the scattering effect is expressed in terms of the transition probability in unit time $W\left(p_{y}, p_{z} \rightarrow p_{y}^{\prime}, p_{z}^{\prime}\right)$ as

$$
\left(\frac{\partial f}{\partial t}\right)_{\text {scatt }}=-\sum_{p_{y}^{\prime}, p_{z}^{\prime}} f(\mathbf{p})\left(1-f\left(\mathbf{p}^{\prime}\right)\right) W\left(p_{y}, p_{z} \rightarrow p_{y}^{\prime}, p_{z}^{\prime}\right)+\sum_{p_{y}^{\prime}, p_{z}^{\prime}} f\left(\mathbf{p}^{\prime}\right)(1-f(\mathbf{p})) W\left(p_{y}^{\prime}, p_{z}^{\prime} \rightarrow p_{y}, p_{z}\right) .
$$

In first order of $\delta f(\mathbf{p})$,

$$
\begin{aligned}
\left(\frac{\partial f}{\partial t}\right)_{\text {scatt }}= & -\sum_{p_{y}^{\prime}, p_{z}^{\prime}} W\left(p_{y}, p_{z} \rightarrow p_{y}^{\prime}, p_{z}^{\prime}\right)\left[\delta f(\mathbf{p})\left(1-f_{0}\left(\varepsilon^{\prime}\right)\right)-f_{0}(\varepsilon) \delta f\left(\mathbf{p}^{\prime}\right)\right] \\
& +\sum_{p_{y}^{\prime}, p_{z}^{\prime}} W\left(p_{y}^{\prime}, p_{z}^{\prime} \rightarrow p_{y}, p_{z}\right)\left[\delta f\left(\mathbf{p}^{\prime}\right)\left(1-f_{0}(\varepsilon)\right)-f_{0}\left(\varepsilon^{\prime}\right) \delta f(\mathbf{p})\right] .
\end{aligned}
$$

Due to the energy conservation, $f_{0}(\varepsilon)=f_{0}\left(\varepsilon^{\prime}\right)$, so we get

$$
\left(\frac{\partial f}{\partial t}\right)_{\text {scatt }}=-\sum_{p_{y}^{\prime}, p_{z}^{\prime}} W\left(p_{y}, p_{z} \rightarrow p_{y}^{\prime}, p_{z}^{\prime}\right)\left[\delta f(\mathbf{p})-\delta f\left(\mathbf{p}^{\prime}\right) .\right] .
$$

Substituting $\delta f(\mathbf{p})=e E v_{z} \tau \frac{\partial f_{0}}{\partial \varepsilon}+\mathscr{O}\left(E^{2}\right)$, we get

$$
\left(\frac{\partial f}{\partial t}\right)_{\text {scatt }}=-e E \frac{\partial f_{0}}{\partial \varepsilon(\mathbf{p})} \sum_{p_{y}^{\prime}, p_{z}^{\prime}} W\left(p_{y}, p_{z} \rightarrow p_{y}^{\prime}, p_{z}^{\prime}\right)\left[\tau(\mathbf{p}) v_{z}(\mathbf{p})-\tau\left(\mathbf{p}^{\prime}\right) v_{z}\left(\mathbf{p}^{\prime}\right)\right] .
$$

in lowest order of $E$. From the eqs.([2.4, $[2.5)$, the left hand side of the above equation is

$$
\left(\frac{\partial f}{\partial t}\right)_{\text {scatt }}=-e E v_{z} \frac{\partial f_{0}}{\partial \varepsilon}+\mathscr{O}\left(E^{2}\right)
$$

Then we get

$$
v_{z}(\mathbf{p})=\sum_{p_{y}^{\prime}, p_{z}^{\prime}} W\left(p_{y}, p_{z} \rightarrow p_{y}^{\prime}, p_{z}^{\prime}\right)\left[\tau(\mathbf{p}) v_{z}(\mathbf{p})-\tau\left(\mathbf{p}^{\prime}\right) v_{z}\left(\mathbf{p}^{\prime}\right)\right]
$$

The interaction between the fermions and the charged impurity is given by the screened Coulomb potential:

$$
V(\mathbf{x})=\left(\frac{4 \pi e^{2}}{\kappa}\right) \frac{\exp \left(-|\mathbf{x}| / r_{0}\right)}{|\mathbf{x}|}
$$

where $r_{0}$ is the screening length, $\kappa$ is dielectric constant.

Transition rate in unit time is given by

$$
W\left(p_{y}, p_{z} \rightarrow p_{y}^{\prime}, p_{z}^{\prime}\right)=2 \pi \sum_{\mathbf{R}}\left|\left\langle p_{y}^{\prime}, p_{z}^{\prime}|v(\mathbf{r}-\mathbf{R})| p_{y}, p_{z}\right\rangle\right|^{2} \delta\left(\omega\left(p_{z}^{\prime}\right)-\omega\left(p_{z}\right)\right),
$$

where $\mathbf{R}$ stands for a position of the impurity. Fourier transform of the screened Coulomb potential is

$$
V(\mathbf{r}-\mathbf{R})=\frac{4 \pi e^{2}}{\kappa} \frac{1}{V} \sum_{\mathbf{q}} \frac{e^{i \mathbf{q} \cdot(\mathbf{r}-\mathbf{R})}}{q^{2}+1 / r_{s}^{2}}
$$


so we should calculati the matrix element $\left\langle p_{y}^{\prime}, p_{z}^{\prime}\left|e^{i \mathbf{q} \cdot \mathbf{x}}\right| p_{y}, p_{z}\right\rangle$. Assuming that the impurities are distributed uniformly with density $N_{I}$, we can obtain the transition rate in unit time

$$
\begin{aligned}
W\left(p_{y}, p_{z} \rightarrow p_{y}^{\prime}, p_{z}^{\prime}\right)= & 2 \pi \delta\left(\omega\left(p_{z}^{\prime}\right)-\omega\left(p_{z}\right)\right)\left(\frac{4 \pi e^{2}}{\kappa}\right)^{2} \frac{N_{I}}{V} \\
& \times \frac{\left[\left(\omega\left(p_{z}^{\prime}\right)+\mu+m\right)\left(\omega\left(p_{z}\right)+\mu+m\right)+p_{z}^{\prime} p_{z}\right]^{2}}{4\left(\omega\left(p_{z}\right)+\mu\right)\left(\omega\left(p_{z}\right)+\mu+m\right)\left(\omega\left(p_{z}^{\prime}\right)+\mu\right)\left(\omega\left(p_{z}^{\prime}\right)+\mu+m\right)} \\
& \times \sum_{q_{x}} \frac{\exp \left[-\frac{1}{2 e B}\left\{q_{x}^{2}+\left(p_{y}-p_{y}^{\prime}\right)^{2}\right\}\right]}{\left[q_{x}^{2}+\left(p_{y}-p_{y}^{\prime}\right)^{2}+\left(p_{z}-p_{z}^{\prime}\right)^{2}+1 / r_{s}^{2}\right]^{2}} .
\end{aligned}
$$

After some calculation, we find

$$
\int d p_{y}^{\prime} d q_{x} \frac{\exp \left[-\frac{1}{2 e B}\left\{q_{x}^{2}+\left(p_{y}-p_{y}^{\prime}\right)^{2}\right\}\right]}{\left[q_{x}^{2}+\left(p_{y}-p_{y}^{\prime}\right)^{2}+\left(p_{z}-p_{z}\right)^{2}+1 / r_{s}^{2}\right]^{2}}=\frac{\pi}{\left(p_{z}-p_{z}^{\prime}\right)^{2}+1 / r_{s}^{2}} I\left(\frac{1}{2 e B}\left(\left(p_{z}-p_{z}^{\prime}\right)^{2}+1 / r_{s}^{2}\right)\right) .
$$

The function $I(\gamma)$ is defined as

$$
I(\gamma) \equiv \int_{0}^{\infty} d x \frac{x e^{-x}}{x+\gamma}=1+\gamma e^{\gamma} \operatorname{Ei}(-\gamma),
$$

where $\operatorname{Ei}(\gamma)$ is the exponential integral. For energy conservation, initial state with $z$ direction momentum $p_{z}$ can be scattered only to final sate with $-p_{z}$. Then eq.(3.14) becomes

$$
\frac{p_{z}}{\varepsilon}=\left(\frac{4 \pi e^{2}}{\kappa}\right)^{2} \frac{N_{I}\left[\left(\varepsilon\left(p_{z}\right)+m\right)^{2}-p_{z}^{2}\right]^{2}}{16 \pi \varepsilon^{2}\left(p_{z}\right)\left(\varepsilon\left(p_{z}\right)+m\right)^{2}} \frac{I\left(\frac{4 p_{z}^{2}+1 / r_{s}^{2}}{2 e B}\right)}{4 p_{z}^{2}+1 / r_{s}^{2}}\left(\tau\left(p_{z}\right)+\tau\left(-p_{z}\right)\right) .
$$

The distribution function is normalized as

$$
\sum_{\mathbf{p}} f(\mathbf{p})=\sum_{\mathbf{p}} f_{0}(\mathbf{p})=1
$$

So the deviation from thermodynamical equilibrium satisfies

$$
\sum_{\mathbf{p}} \delta f(\mathbf{p})=0
$$

At zero temperature

$$
\delta f(\mathbf{p})=e E \tau(\mathbf{p}) \delta(\varepsilon-\mu) \frac{p_{z}}{\varepsilon}
$$

therefore we get

$$
\sum_{p_{x}, p_{y}}\left(\tau\left(p_{x}, p_{y}, p_{z}\right)-\tau\left(p_{x}, p_{y},-p_{z}\right)\right)=0 .
$$

Then the relaxation time is

$$
\frac{1}{\tau\left(p_{z}\right)}=\left(\frac{4 \pi e^{2}}{\kappa}\right)^{2} \frac{N_{I}\left[\left(\varepsilon\left(p_{z}\right)+m\right)^{2}-p_{z}^{2}\right]^{2}}{8 \pi p_{z} \varepsilon\left(p_{z}\right)\left(\varepsilon\left(p_{z}\right)+m\right)^{2}} \frac{I\left(\frac{4 p_{z}^{2}+1 / r_{s}^{2}}{2 e B}\right)}{4 p_{z}^{2}+1 / r_{s}^{2}}
$$

Only the states with $\varepsilon=\mu$ contribute the scattering, so we substitute $p_{z}=\sqrt{\mu^{2}-m^{2}}$. Then

$$
\frac{1}{\tau}=\frac{8 \pi e^{4} N_{I}}{\kappa^{2}} \frac{m^{2}}{\mu \sqrt{\mu^{2}-m^{2}}} \frac{1}{4\left(\mu^{2}-m^{2}\right)+1 / r_{s}^{2}} I\left(\frac{1}{2 e B}\left(4\left(\mu^{2}-m^{2}\right)+1 / r_{s}^{2}\right)\right) .
$$

This result matches with the result in [四] in non-relativistic limit. 


\section{Summary and discussion}

In this report, we have presented our study on relaxation time for the relativistic fermions scattered by the Coulomb impurities in strong magnetic field. We reviewed the basics of the transport theory. Calculating transition probability, we have obtained the relativistic expression for the relaxation time.

In strong magnetic field limit $e B \rightarrow \infty, I\left(\frac{1}{2 e B}\left(4\left(\mu^{2}-m^{2}\right)+1 / r_{s}^{2}\right)\right) \rightarrow 1$, then

$$
\frac{1}{\tau} \rightarrow \frac{8 \pi e^{4} N_{I}}{\kappa^{2}} \frac{m^{2}}{\mu \sqrt{\mu^{2}-m^{2}}} \frac{1}{4\left(\mu^{2}-m^{2}\right)+1 / r_{s}^{2}} .
$$

Then we get the interpolating formula between the relativistic and non-relativistic relaxation time in strong magnetic field limit:

$$
\begin{aligned}
& \frac{1}{\tau} \propto m^{2} \quad(m / \mu \sim 0) \\
& \frac{1}{\tau} \propto\left(\mu^{2}-m^{2}\right)^{-1 / 2} \quad(m / \mu \sim 1) .
\end{aligned}
$$

In our study, we consider only the lowest band. When $2 e B<p_{z}^{2}+m^{2}-\mu^{2}$, the first excited states come to contribute the scattering. So we should consider higher energy band states in that case. We considered zero temperature case where scattering by the acoustic phonon can be neglected. However in finite temperature case, we should calculate the effect of the phonon scattering.

\section{References}

[1] P. N. Argyres and E. N. Adams, Longitudinal magnetoresistance in the quantum limit, Phys. Rev. 104 (Nov, 1956) 900-908.

[2] H. B. Nielsen and M. Ninomiya, ADLER-BELL-JACKIW ANOMALY AND WEYL FERMIONS IN CRYSTAL, PhyS. Lett. BI30(1983) 389.

[3] X. Wan, A. M. Turner, A. Vishwanath and S. Y. Savrasov, Topological semimetal and fermi-arc surface states in the electronic structure of pyrochlore iridates, Phys. Rev. B 83 (May, 2011) 205101.

[4] Q. Li, D. E. Kharzeev, C. Zhang, Y. Huang, I. Pletikosic, A. V. Fedorov et al., Observation of the chiral magnetic effect in ZrTe5, Nature Phys. 12 (2016) 550-554, [11412. 6543].

[5] X. Huang, L. Zhao, Y. Long, P. Wang, D. Chen, Z. Yang et al., Observation of the chiral-anomaly-induced negative magnetoresistance in $3 d$ weyl semimetal taas, Phys. Rev. X 5 (Aug. 2015) 031023 .

[6] C. Zhang et al., Observation of the Adler-Bell-Jackiw chiral anomaly in a Weyl semimetal, 1503.02630 .

[7] C. Zhang, E. Zhang, Y. Liu, Z.-G. Chen, S. Liang, J. Cao et al., Detection of chiral anomaly and valley transport in Dirac semimetals, ArXiv e-prints (Apr., 2015),

[8] J. Xiong, S. K. Kushwaha, T. Liang, J. W. Krizan, W. Wang, R. J. Cava et al., Signature of the chiral anomaly in a Dirac semimetal: a current plume steered by a magnetic field, ArXiv e-prints (Mar., 2015), [1503.08179]. 